\title{
Organização didático pedagógica - estratégias para o desenvolvimento de competências
}

Luhana Santos Gonzales Garcia*; Jussara Lança Lopes**; Beatriz Sartori da Silva***; Elisa Emi Tanaka****

\footnotetext{
* Doutoranda em Saúde Bucal da Criança, UNESP Estudante do Curso de Odontologia, UEL

*** Residente em Odontopediatria, UEM

**** Chefe do Departamento de Medicina Oral e Odontologia Infantil da UEL
}

\section{RESUMO}

As Diretrizes Curriculares Nacionais (DCN) para o curso de graduação em Odontologia foram instituídas no ano de 2002 reorientando o ensino na direção da formação autônoma, pautada em princípios éticos e humanísticos, crítico-reflexiva, atuação em diferentes níveis de atenção e adequando-o às demandas sociais. Para alcançar estes objetivos a organização e metodologias tradicionais não têm se mostrado suficientes e novas estratégias pedagógicas têm sido propostas. Neste contexto, o objetivo do trabalho foi analisar o Projeto Pedagógico do Curso (PPC) de Odontologia das Instituições de Ensino Superior (IES) públicas e privadas do Brasil, com o propósito de caracterizar e analisar a estrutura curricular dos cursos. Foram pesquisadas 203 IES cadastradas no sistema de tramitação eletrônica de processos regulatórios das IES (e-MEC). Apenas 36 disponibilizavam integralmente os PPC na internet. $89 \%$ distribuem os conteúdos por disciplinas e $11 \%$ por módulos. Dos cursos que estão organizados por disciplinas, $53 \%$ agrupam-nas por áreas, eixos ou ciclos, mas sem demonstrar como acontece a integração dos conteúdos. Conclui-se que as instituições, mesmo após a realização das reformas curriculares, ainda necessitam realizar mudanças significativas para alcançar os objetivos das DCN.

Descritores: Educação em Odontologia. Currículo. Instituições de Ensino Superior. Educação Baseada em Competências. Ensino.

\section{INTRODUÇÃO}

As Diretrizes Curriculares Nacionais $(\mathrm{DCN})^{1}$ para o curso de graduação em Odontologia foram instituídas no ano de 2002, reorientando o ensino na direção da formação pautada em princípios éticos e humanísticos, na capacidade crítico-reflexiva, atuação em diferentes níveis de atenção e adequada às demandas sociais ${ }^{2}$.

Essa nova proposta curricular proporciona um ensino baseado no desenvolvimento de competências, que podem ser descritas como a capacidade de mobilizar conhecimentos nas diferentes áreas ${ }^{3}$ para que ocorra a solução dos problemas encontrados na prática cotidiana, com enfoque 
na resolução em nível individual e coletivo de forma multidisciplinar ${ }^{4}$.

O Projeto Pedagógico do Curso (PPC) não pode ser entendido como uma simples proposição de disciplinas e conteúdos, sem que as mesmas se articulem em torno de estratégias amplas em um projeto global com objetivos claros de seu objeto de formação (o profissional) vinculado com a realidade local $^{3,5}$.

$\mathrm{O}$ ato de ensinar-aprender deve ser um conjunto de atividades articuladas, nas quais professor e aluno compartilham, cada vez mais, parcelas de responsabilidade e comprometimento visando à aprendizagem significativa ${ }^{6}$.

Considerando o pensamento de Ausubel $^{7}$ e Morin $^{8}$, para que ocorra aprendizagem de forma significativa, a teoria deve estar vinculada com a prática e os conteúdos devem estar interligados e possuir significado para que o aluno se sinta motivado a aprender e apreender os conhecimentos.

Assumir esse novo modelo na formação de profissionais de saúde com perfil generalista, humanista, crítico reflexivo, para atuar em todos os níveis de atenção à saúde ${ }^{1}$, implica no enfrentamento de novos desafios ${ }^{9}$.

O ponto de partida é a definição do perfil, seguido dos objetivos do Curso e então propostas de estratégias de organização curricular. Em uma delas, encontram-se elementos que funcionarão como eixos em fases específicas (eixo horizontal) ou ao longo de todo o curso (eixo vertical).

Estes eixos são elementos centrais, sobre os quais definem-se e articulam-se os saberes, os conceitos, princípios, leis, quadros teórico-práticos, visando superar a forma estanque tradicionalmente presente nas grades curriculares $^{10}$.

Neste contexto, este trabalho teve como objetivo analisar os PPC das Instituições de Ensino Superior (IES) de Odontologia do Brasil, com o propósito de caracterizar e analisar a estrutura curricular dos mesmos verificando suas estratégias e consequentemente o atendimento às DCN.

\section{METODOLOGIA}

Foi realizado o levantamento de dados das Instituições de Ensino Superior de Odontologia, públicas e privadas, cadastradas no Sistema Eletrônico de Acompanhamento dos Processos que Regulam a Educação Superior no Brasil (e-MEC), no ano de 2014. A partir destes dados, iniciou-se a busca, nos sites das instituições, que disponibilizavam o PPC do curso na internet, tendo como critério de inclusão apenas as IES que apresentavam o PPC na íntegra.

As informações obtidas foram transcritas para uma planilha no Excel® 2010 (Microsoft Co., Redmond, Washington, EUA). Os dados numéricos foram analisados por meio de estatística descritiva.

Trata-se de um estudo transversal, quantitativo, com dados secundários disponibilizados nos sítios eletrônicos das IES que oferecem curso de Odontologia.

Foram realizadas leituras detalhadas de cada um dos projetos buscando informações sobre as organizações curriculares, distribuiçõos dos conteúdos e estágios supervisionados.

Por ser uma pesquisa que utilizou dados de caráter público, foi solicitada a isenção da aprovação do Comitê de Ética em Pesquisa.

\section{RESULTADOS}

Do total de 203 IES pesquisadas, cadastradas no e-MEC no ano de 2014, 36 
disponibilizam PPC na rede, o que representa $18 \%$ do total de IES elegíveis para o estudo.

Das IES selecionadas (36 IES), 22 (61\%) são instituições públicas e 14 (39\%) instituições privadas.

Quanto à organização dos conteúdos na matriz curricular, $89 \%$ das instituições distribuem os conteúdos por disciplinas e $11 \%$ por módulos disciplinares.

Das 36 IES, 34 (89\%) distribuem os conteúdos por disciplinas, $17(53 \%)$ as agrupam por Área de Conhecimento, 7 IES (22\%) por Eixos de Formação, 5 IES (16\%) por Ciclos de Formação, 2 IES (6\%) por Conteúdo de Estudo, 1 IES (3\%) por Eixos Temáticos, 1 IES (3\%) por Conjunto Instrucional, 1 IES (3\%), conforme o gráfico 1.

Em relação aos estágios supervisionados extramuros, $89 \%$ das instituições os apresentaram nos seus PPC e $11 \%$ não os mencionaram.

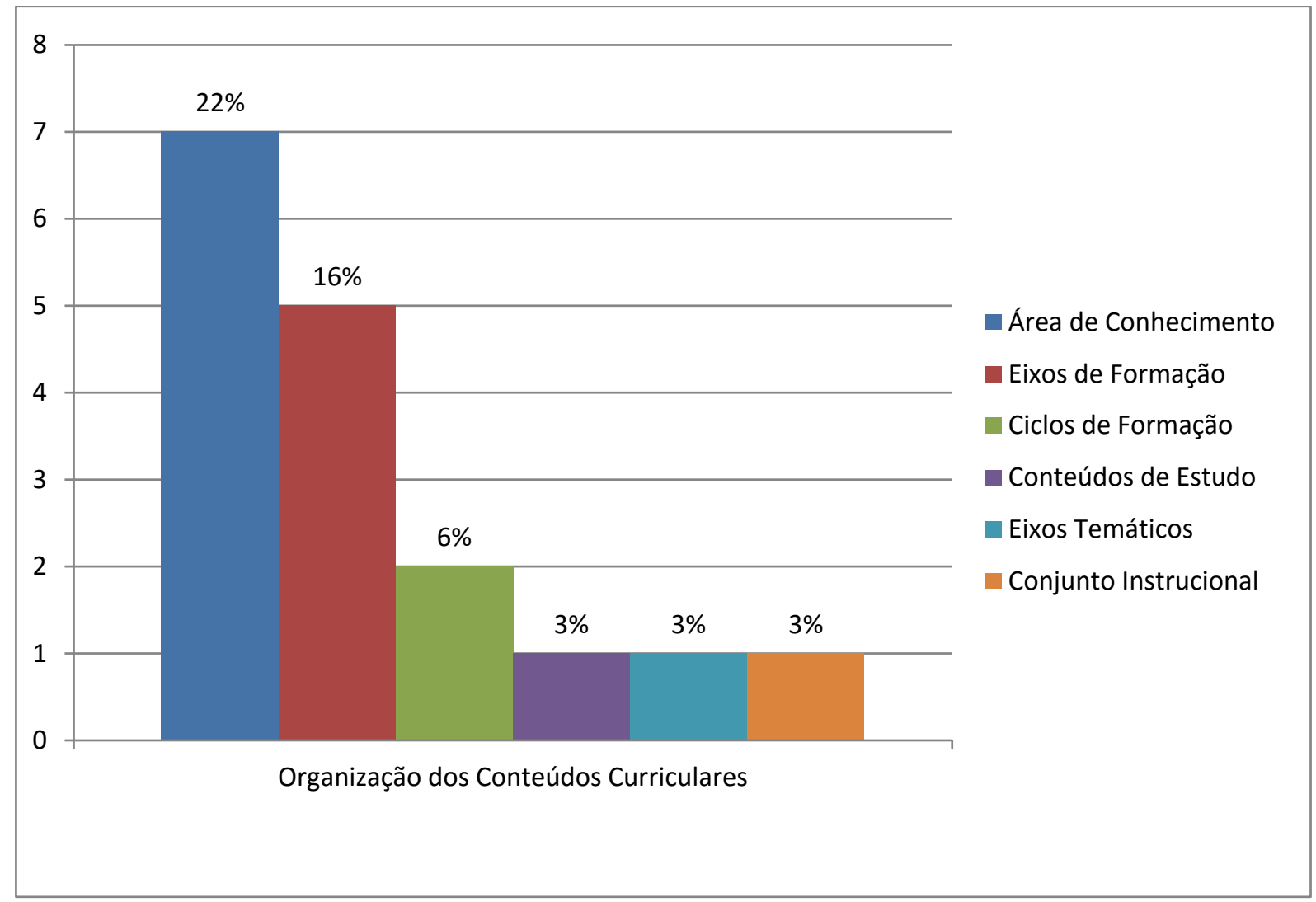

Gráfico 1. Organização dos conteúdos por unidades de estudo.

\section{DISCUSSÃO}

Segundo as $\mathrm{DCN}^{1}$, a organização curricular deve "aproximar o conhecimento básico da sua utilização clínica; viabilização pela integração curricular; utilizar metodologias de ensino/aprendizagem que permitam a participação ativa dos alunos neste processo e a integração dos conhecimentos das ciências básicas com os das ciências clínicas e, instituir programas de iniciação científica como método de aprendizagem." 
Observa-se que quanto à organização dos conteúdos na matriz curricular, $89 \%$ das instituições distribuem os conteúdos por disciplinas, e apenas $11 \%$ estruturam os conteúdos por módulos e estes constituem-se num espaço responsável por atividades ou temas definidos, com tarefas e componentes articulados ao currículo.

Dos cursos que organizam seus conteúdos por disciplinas, 53\% agrupam-nas por Área de Conhecimento, Eixos de Formação, Ciclos de Formação, Conteúdo de Estudo, Eixos Temáticos, Conjunto Instrucional, mas nenhuma demonstrou como acontecerá a integração dos conteúdos entre as disciplinas para a formação. Foi observado apenas a diferenciação de nomenclatura, no entanto a aplicação é a mesma.

As DCN orientam que os conteúdos essenciais devem contemplar: Ciências Biológicas e da Saúde, Ciências Humanas e Sociais e Ciências Odontológicas. Não especificando que estes devem ser os eixos orientadores do Curso como observado em 23 PPC. E são claras em orientar que estes conteúdos devem estar relacionados com todo o processo saúde-doença do cidadão, da família e da comunidade, integrado à realidade epidemiológica e profissional. Segundo Morita e Kriger, $2004^{11}$, deve-se ter o cuidado ao interpretar as DCN, pois em um primeiro momento podem dar a impressão de serem redundantes e abstratas, apontando para atividades que já estão presentes nos projetos pedagógicos tradicionais dos cursos de Odontologia, mas na verdade apontam para uma necessidade de transformação substantiva dos mesmos e não apenas uma acomodação de situações existentes.

Os outros $36 \%$, se estruturam na forma de disciplinas separadas por áreas básicas, áreas profissionalizantes e os estágios ao final do curso. Estes modelos de ensino são uma combinação dos Modelos Jesuítico e Napoleônico ${ }^{4}$. O Jesuítico, proposto há 500 anos, é um modelo onde o conhecimento é tomado como algo posto, indiscutível, pronto e acabado, passado do professor para o estudante por meio de aulas expositivas teóricas, usando a memorização como exercício essencial do processo de aprendizagem. $\mathrm{O}$ modelo francêsnapoleônico, de 1808, é voltado para o ensino profissionalizante, utiliza a teoria como base para a prática e o currículo é dividido em ciclo básico, ciclo profissionalizante e, ao final do curso, os estágios.

A organização por eixos é uma forma de estruturação curricular integradora dos conhecimentos a ser proposto ${ }^{10}$. Podendo funcionar nos currículos como eixo vertical, o qual integra, ordena e orienta os conteúdos no decorrer do curso, perpassando por todas as séries, de maneira que os conhecimentos adquiridos no início do processo constituam embasamento para os anos subsequentes; e o eixo horizontal, organiza o relacionamento entre os conteúdos dentro de um mesmo semestre ou série ${ }^{10}$.

Esses conteúdos podem ser distribuídos ao longo do curso de maneira integradora, em fases específicas, ou ao longo de todo o curso, visando à aprendizagem do aluno, tornando-a significativa, não apenas memorização de procedimentos estanques. Podem estar agrupados e inter-relacionados por meio de temas e assuntos comuns a todas às unidades de ensino, sejam elas módulos ou disciplinas.

Desta forma foi possível observar que a articulação do eixo na estrutura curricular de um dos projetos, o qual apresenta um eixo temático central norteador, que se secciona em 10 segmentos transversais para favorecer a 
formação profissional. E o eixo curricular central que se divide em dois segmentos, o primeiro segmento designa a Unidade Integrada de Prática Interdisciplinar, tendo a finalidade habilitar os estudantes cientificamente com aproximação inicial entre os diversos conteúdos disciplinares (ciências biológicas da saúde, odontológica e as disciplinas complementares obrigatórias). No segundo segmento, designa Unidade Integrada de Atuação Interdisciplinar, tendo como finalidade manter aproximação com os conteúdos disciplinares, conhecer a realidade da população, visando o cuidado e a assistência odontológica humanizada.

Neste projeto está demonstrado o eixo como o objeto central que irá nortear a integração das disciplinas e seus conteúdos.

Verificou-se em outro projeto a utilização de disciplinas distribuídas ao longo do curso como estratégias integradoras. As disciplinas de Seminário de Integração, o qual tem como objetivo estimular os estudantes a alcançar a articulação do conhecimento obtidos nas diferentes áreas ministradas separadamente. Essas disciplinas estão presentes na estrutura curricular do $1^{\circ}$ ao $4^{\circ}$ semestre tem como objetivo fomentar as discussões sobre casos clínicos e seu significado no processo saúde doença nas formações sociais. Desta forma observa-se o incentivo ao ensino baseado no desenvolvimento de competências, aprendizagem significativa, o aluno como sujeito ativo, responsável pela própria aprendizagem $^{12}$.

Em 7 projetos encontraram estratégias pedagógicas de mudanças no currículo com objetivo de alcançar as DCN, contemplando a interdisciplinaridade e a flexibilização do currículo. Criaram-se as macro disciplinas, em ordem de complexidade crescente evitando as antigas disciplinas "estanques" separadas pelas especialidades. Além disso, utilizam estratégias de aprendizagem com dinâmicas de grupo, seminários, encontros, demonstrando a preocupação constante com a efetiva participação do aluno em sala de aula, quer seja ela teórica, laboratorial ou clínica. A partir dessas estratégias, é observada a tentativa da transformação para um currículo integrador.

Em alguns PPC apenas há denominação de eixos e descrição de conteúdos, mas sem apresentação de estratégias de operacionalização ou de que maneira os componentes deste eixo (disciplinas) se relacionam com os conteúdos para alcançar o objetivo da formação.

A divisão dos conteúdos por disciplina é uma possibilidade prevista nas DCN, porém, para que o PPC esteja de acordo com as DCN, deve atender ao pressuposto do ensino integrado, com uma proposta globalizada, tendo ao centro o estudante, preparando-o para atuar em todos os níveis de atenção à saúde $^{6}$, consequentemente é preciso ficar claro no PPC de que forma isso ocorrerá.

Analisando os projetos, todos os cursos apresentam seu currículo como integrado seguindo as DCN, mas observa-se que em $64 \%$ dos projetos, a integração dos conteúdos só ocorre nos últimos anos, principalmente $4^{\mathrm{a}}$ e $5^{\mathrm{a}}$ série, nas denominadas clínicas integradas e nas atividades de estágio supervisionado.

Em um dos currículos, a IES descreve que à medida que o estudante vai adquirindo competência nas clínicas estanques, o desenvolvimento mais intenso será realizado nas clínicas integradas. Desta forma, seguindo ainda a configuração em grade, mantem a separação da teoria e prática e as disciplinas do básico e do profissionalizante. 
Este modelo de projeto não faz com que o estudante construa um quadro teóricoprático global significativo mais próximo da realidade profissional, na qual atuará depois de concluída a graduação ${ }^{8,9}$.

Os estágios supervisionados extramuros são atividades que possibilitam ao estudante vivenciar a realidade em um ambiente externo, no qual seu papel é social, coloca-se frente aos desafios da população ${ }^{13,14}$. Está é uma atividade obrigatória nos PPC apontada nas DCN com carga horária mínima de $20 \%$ da carga horária total do curso. Segundo Morita e Kriger ${ }^{11}$, uma das competências a ser desenvolvida neste cenário é a capacidade de liderança, a qual necessita de algumas habilidades que só podem ser desenvolvidas em oportunidades práticas. Não basta dizer que se espera que o profissional de Odontologia seja capaz de assumir a liderança na equipe de saúde. Ele tem que ter tido durante a sua formação oportunidades concretas para desenvolver esse papel além de estar inserido na realidade lhe traz maiores subsídios para as tomadas de decisão.

Das 36 IES pesquisadas, 11\% não apresentaram nos seus PPC os estágios supervisionados extramuros. As instituições reconhecem a importância desses estágios para a formação profissional, através dos mesmos os estudantes têm a oportunidade de conhecer o Sistema Único de Saúde (SUS), integrando-se com outros profissionais da área da saúde. Colocando-os em contato direto com os problemas reais da saúde pública, possibilitando a formação de um profissional comprometido.

Por meio das organizações dos PPC, foi possível observar que os cursos estão buscando alcançar os objetivos das DCN com atividades interdisciplinares na tentativa de aproximação dos conhecimentos básicos e profissionalizantes, mas muitos deles apenas utilizam termos teóricos sem demonstração de estratégias, não evidenciaram como chegar ao seu real objetivo: a formação de um profissional generalista, humanista, crítico e reflexivo, para atuar em todos os níveis de atenção à saúde. Esta organização segue ainda os modelos Jesuítico e Napoleônico, com disciplinas isoladas, teoria separada da prática. Esses modelos não permitem que ocorra aprendizagem de forma significativa, não atingindo os objetivos do desenvolvimento de competências.

\section{CONCLUSÃO}

Conclui-se que os cursos analisados estão buscando atender às DCN, mas precisam rever suas estratégias pedagógicas, visando um ensino integrador, centrada no educando, realmente preparando-o para atuar em todos os níveis da atenção a saúde.

Os PPC analisados encontram-se estruturados por disciplinas e módulos, porém a integração da formação fica restrita a poucos deles.

\section{ABSTRACT \\ Educational teaching organization - strategies to skills development}

The National Curriculum Guidelines (DCN) for the Dentistry course were introduced in 2002 in order to reorient teaching toward autonomous education, guided by ethical, humanistic, critical and reflexive principles, acting upon different levels of care and adapting it the social demands. The traditional methodologies and organization have not proven to be sufficient in order to reaching such objectives, and new educational strategies have emerged. In such context, this study was aimed at analyzing the Educational Project of the Course (EPC) of the Institutions of Higher Education (IHE) from Brazilian 
public and private Dentistry courses, characterizing and analyzing their Syllabus. From 203 IHE registered in the electronic processing system that regulates Brazilian IHE (e-MEC), only 36 completely provided EPC on the Internet, $89 \%$ distributed the contents for subjects and $11 \%$ for modules. Among the courses that are organized by subjects, $53 \%$ used to group them by areas, axes, or cycles, but without showing how the integration of contents occur. It can be inferred that the Institutions, even after the implementation of Syllabus changes, still need to make notorious shifts in order to achieve the DCN goals.

Descriptors: Education, Dental. Curriculum. Higher Education Institutions. CompetencyBased Education. Education.

\section{REFERÊNCIAS}

1. Brasil. LDB: Lei de Diretrizes e Bases da Educação Nacional. Lei no 9.394 de 20 de dezembro de 1996, que estabelece as diretrizes e bases da educação nacional. Diário Oficial da União, Brasília, 23 de dezembro de 1996, seção 1, p. 27833 a 27841. [Acesso em: 26/11/2014] Disponível em: http://portal.mec.gov.br /seed/arquivos/pdf/tvescola/leis/lein9394. pdf

2. Brasil. Conselho Nacional de Educação. Câmara de Educação Superior. Resolução CNE/CES 3, de 19 de fevereiro de 2002. Institui Diretrizes Curriculares Nacionais do Curso de Graduação em Odontologia. Diário Oficial União, seção1:10, 04 mar 2002. [Acesso em: 26/11/2014] Disponível em: $\quad$ http://portal.mec.gov.br /cne/arquivos/pdf/CES032002.pdf

3. Mitre SM, Siqueira-Batista R, Girardi-de-
Mendonça JG, Morais-Pinto NM, Meirelles CAB, Pinto-Porto $\mathrm{C}$, et al. Metodologias ativas de ensinoaprendizagem na formação profissional em saúde: debates atuais. Ciênc Saúde Coletiva. 2008; 13(sup. 2):2133-44.

4. Anastasiou LGC. Temas e textos da Educação Superior - Metodologia de ensino na universidade brasileira: elementos de uma trajetória. Campinas: Papirus, 2001.

5. Morita MC, Kriger L, Perri de Carvalho AC, Haddad AE. Implantação das Diretrizes Curriculares Nacionais em Odontologia. 2 ed., Maringá: Dental Press, ABENO, OPAS, MS, 2013.

6. Albuquerque VS, Batista RS, Tanji S, Moço ETSM. Currículos disciplinares na área de saúde: ensaio sobre saber e poder. Interface. 2009; 13(31):261-72.

7. Ausubel DP, Novak, JD, Hanesian H. Educational Psychology: A Cognitive View. 2ed., New York: Holt, Rinehart and Winston, 1978.

8. Morin E. A cabeça bem-feita, repensar a reforma, reformar o pensamento. 8 ed., Rio de Janeiro: Bertrand Brasil, 2003, 128p.

9. Ceccim $\mathrm{R}$ B, Feuerwerker LCM. Mudanças na graduação das profissões de saúde sob eixo da integralidade. Cad Saúde Públ. 2004; 20(5):1400-10.

10. Anastasiou LGC, Alves LP. Processos de aprendizagem na universidade: pressupostos para as estratégias de trabalho em aula. 10ed., Joinville, SC: UNIVILLE, 2012.

11. Morita MC, Kriger L. Mudanças nos cursos de odontologia e a interação com o SUS. Rev ABENO.; 2004, 4(1):17-21.

12. Araujo, ME. Palavra e silêncio na educação superior em odontologia, Ciênc Saúde Coletiva. 2006; 11(1):179-82. 
13. Associação Brasileira de Ensino Correspondência para:

Odontológico. Diretrizes da ABENO para Elisa Emi Tanaka

a definição do estágio supervisionado nos e-mail: elisatanaka@ hotmail.com

cursos de Odontologia. Rev ABENO. Departamento de Medicina Oral e 2002; 2(1):39.

14. Werneck MAF, Senna MIB, Drumond Odontologia Infantil da UEL

Rua Pernambuco, 540 - Centro

MM, Lucas SD. Nem tudo é estágio: 86020-120 Londrina/PR

Contribuições para o debate. Ciênc Saúde

Coletiva. 2010;15(1):221-31. 